\title{
Erratum: Effect of Photon Energy on Conventional Intensity-Modulated Radiotherapy and Rapid Arc Radiotherapy Planning for Deep-Seated Targets in Carcinoma Cervix
}

\author{
Manindra Bhushan ${ }^{1,2}$ Girigesh Yadav ${ }^{1}$ Deepak Tripathi ${ }^{2} \quad$ Lalit Kumar $^{1,3}$ Abhinav Dewan ${ }^{1}$ \\ Inderjit Kaur Wahi ${ }^{1}$ Mahamood Suhail ${ }^{1}$ Swarupa Mitra ${ }^{1} \quad$ Munish Gairola ${ }^{1}$
}

\footnotetext{
${ }^{1}$ Division of Medical Physics and Department of Radiation Oncology, Rajiv Gandhi Cancer Institute and Research Centre, New Delhi, India

${ }^{2}$ Amity School of Applied Sciences, Amity University (AUUP), Noida, Uttar Pradesh, India

${ }^{3}$ Dr. APJ Abdul Kalam Technical University, Lucknow, Uttar Pradesh, India
}

Asian J Oncol

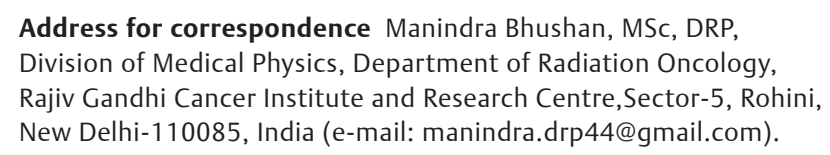
Division of Medical Physics, Department of Radiation Oncology, Rajiv Gandhi Cancer Institute and Research Centre,Sector-5, Rohini, New Delhi-110085, India (e-mail: manindra.drp44@gmail.com).

\section{Erratum}

It has been brought to the Publisher's attention that - Figures $\mathbf{2}$ and $\mathbf{3}$ were published incorrectly in the above article published in Asian Journal of Oncology (DOI: 10.1055/s0039-1693523) on July 19, 2019. 
The correct figures with their legends appear as below:
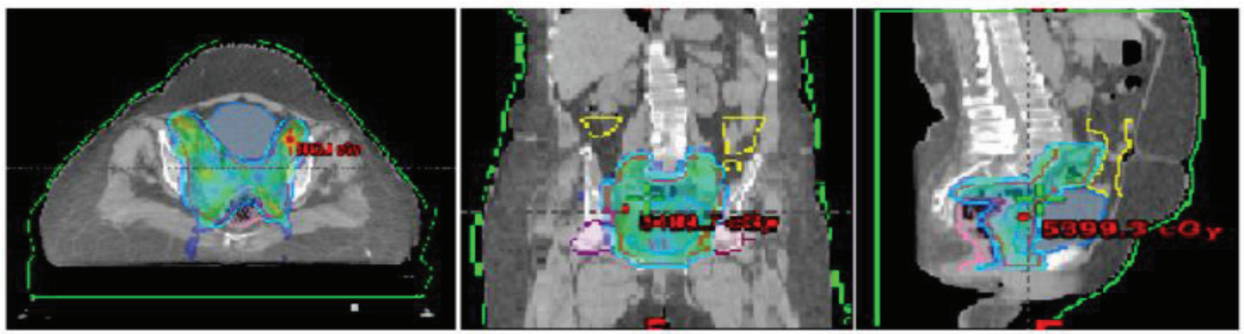

IMRT 6MV
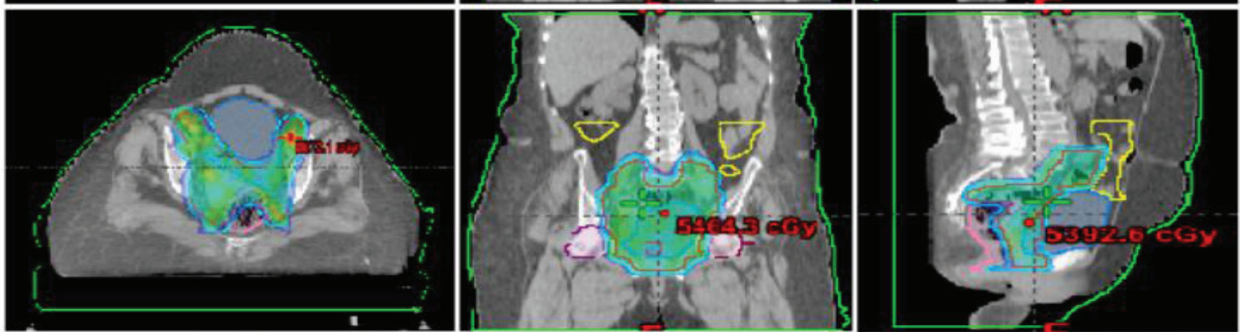

IMRT $10 \mathrm{MV}$
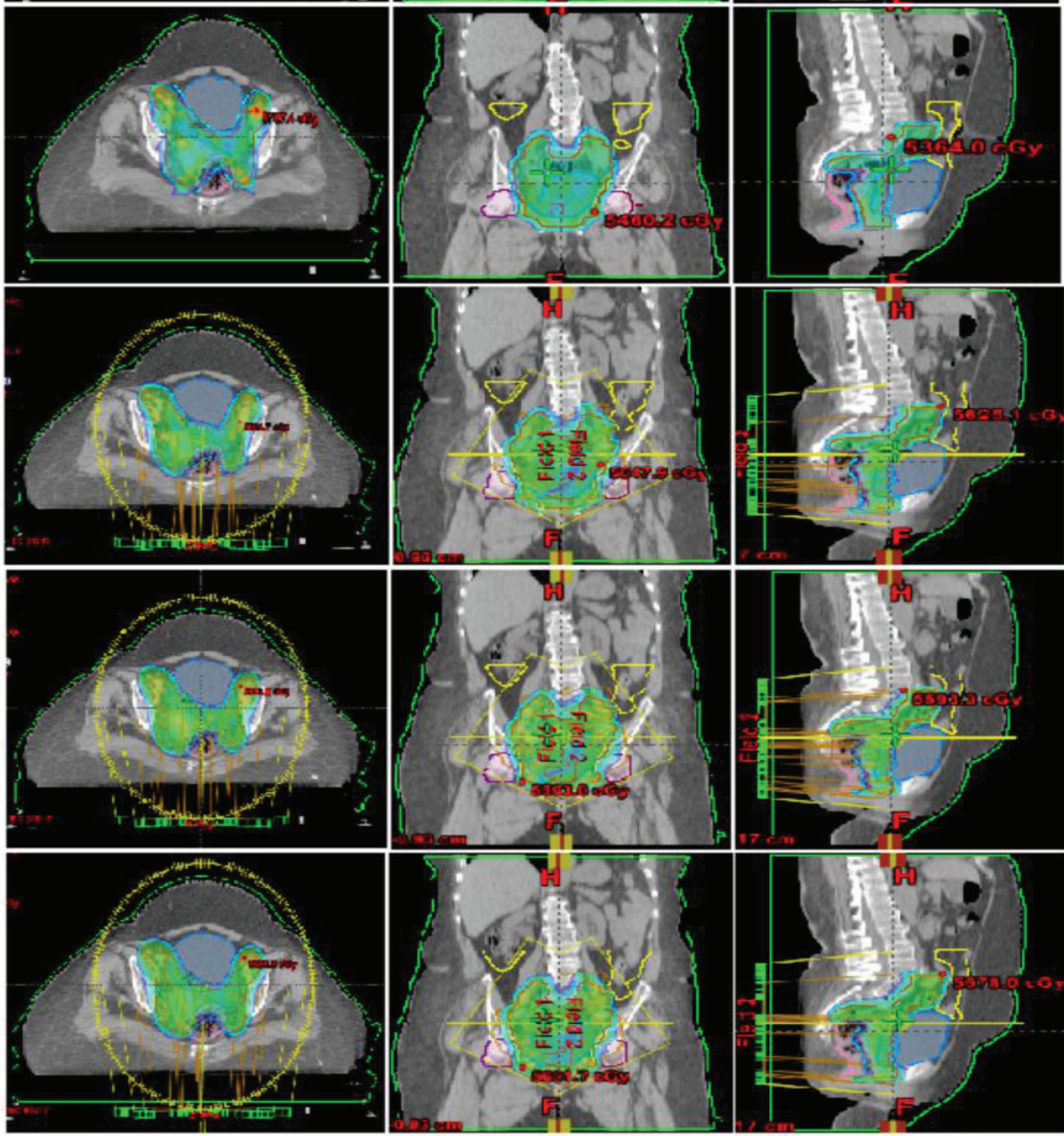

AXIAL VIEW



CORONAL VIEW

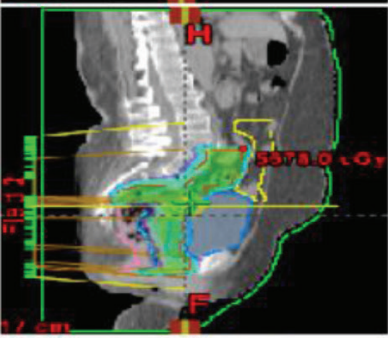

SAGITTAL VIEW

IMRT $15 \mathrm{MV}$

\section{DA $6 \mathrm{MV}$}

DA $10 \mathrm{MV}$

DA $15 \mathrm{MV}$

Fig. 2 Dose coverage of low dose regions (28 Gy) for 6 MV, 10 MV, and 15 MV for IMRT and dual arc (DA) plans. IMRT, intensity-modulated radiotherapy. 

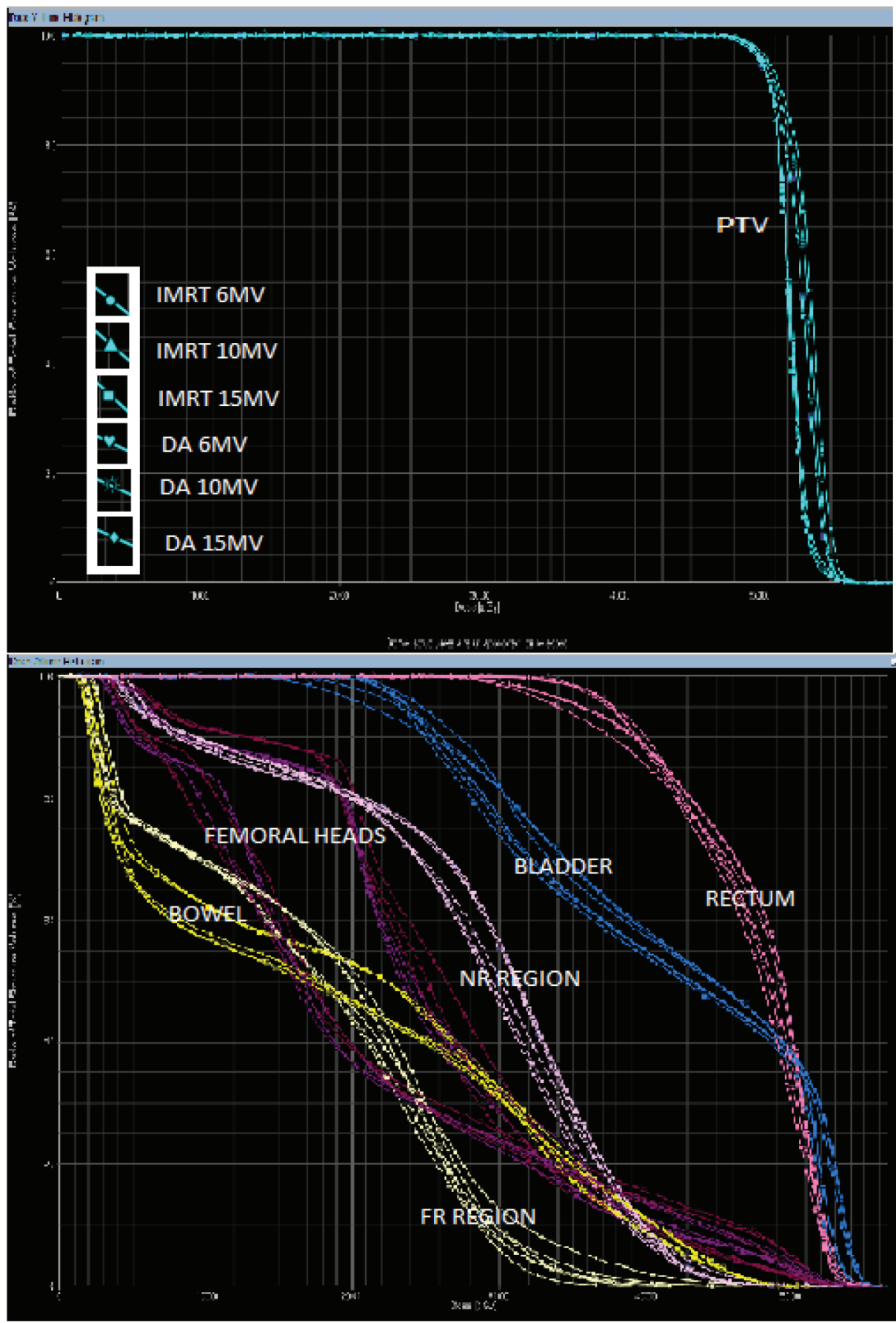

Fig. 3 Cumulative dose volume histogram (DVH) of PTV and OARs. PTV, planning target volume; OARs, organs at risk. 